\title{
TSC1 Gene Inactivation
}

National Cancer Institute

\section{Source}

National Cancer Institute. TSC1 Gene Inactivation. NCI Thesaurus. Code C40462.

A mutation in the TSC1 gene that either inhibits the expression of or results in the translation of an inactive hamartin protein. 